\title{
Synthesis of Positive-Real Functions with Low-Complexity Series-Parallel Networks
}

\author{
Jason Zheng Jiang and Malcolm C. Smith
}

\begin{abstract}
The purpose of this paper is to continue to develop the recently introduced concept of a regular positivereal function and its application to the classification of lowcomplexity two-terminal networks. This paper studies five- and six-element series-parallel networks with three reactive elements and presents a complete characterisation and graphical representation of the realisability conditions for these networks. The results are motivated by an approach to passive mechanical control which makes use of the inerter device.
\end{abstract}

\section{INTRODUCTION}

A famous theorem in electrical networks by Bott and Duffin [1] showed that any positive-real function could be realised as the driving-point immittance of a network consisting of resistors, capacitors and inductors only. The apparent non-minimality of the construction has subsequently intrigued many researchers and there were a number of important papers which followed up on this question, e.g. [2], [3], [4], [5]. Interest in the topic lost momentum in the early 1970s due to the growing importance of integrated circuits.

Recently, a new network element (the inerter) was introduced for mechanical control [6] which has revived interest in passive network realisations. The inerter is a mechanical two-terminal element with the property that the applied force at the terminals is proportional to the relative acceleration across the terminals. Applications of the method to vehicle suspension [7], [8], control of motorcycle steering instabilities [9], [10] and vibration absorption [6] have been identified. The inerter has been successfully deployed in Formula One racing since 2005 [11].

For mechanical realisations, minimising network complexity is important. As such, there is fresh motivation for a systematic classification of the realisability conditions of simple networks. Within the electrical circuit literature, alongside the powerful and general synthesis results of Cauer, Foster, Brune, Bott-Duffin, Darlington, there was a long-running attempt to classify the realisability condition for simple networks by means of enumeration [12], [13], [14], [15], [16], [17], [18], [19], [20], [21], [22]. Many partial results were established but a complete picture was never obtained, even for the apparently simple case of a biquadratic.

The present paper is a successor to [23] which formalised the concept of a regular positive real function, introduced the terminology of a network quartet, and gave a complete reworking and characterisation of the class of transformerless networks containing two reactive elements. The present paper considers five- and six-element series-parallel networks with

Both authors are with the Department of Engineering, University of Cambridge, Cambridge CB2 1PZ, U.K.; zj219@cam.ac.uk, mcs@eng.cam.ac.uk three reactive elements which draws on and reworks results of Foster, Ladenheim, Vasiliu, Reichert and others [24], [25]. Among the networks with three reactive elements it is verified that there is one network quartet with five elements and four network quartets with six elements which may realise non-regular positive-real biquadratic functions. In each case, the non-regular realisable regions for a biquadratic function in canonical form are determined. It is shown that the non-regular realisable regions for three of the six-element network quartets are identical and have a boundary which coincides with the realisability curve for the five-element quartet. The fourth six-element quartet is shown to realise a different non-regular region from the other quartets.

\section{Regular Positive-Real Functions}

In this section we recall the concept of regularity and its properties given in [23].

Definition: A positive-real function $Z(s)$ is defined to be regular if the smallest value of $\operatorname{Re}(Z(j \omega))$ or $\operatorname{Re}\left(Z^{-1}(j \omega)\right)$ occurs at $\omega=0$ or $\omega=\infty$.

Lemma 1: Let $Z(s)$ be a regular positive-real function. Then $\alpha Z(s), Z(\beta s), Z\left(s^{-1}\right), Z^{-1}(s)$ are all regular, where $\alpha, \beta>0$.

Lemma 2: Let $Z(s)$ be a regular positive-real function. Then $Z(s)+R$ and $Z^{-1}(s)+R^{-1}$ are both regular, where $R$ is nonnegative.

Lemmas 1 and 2 imply that if a network can only realise regular immittances, then so will the dual network (if it exists), the network obtained by replacing inductors with capacitors of reciprocal values (and vice versa) and the network obtained by adding a resistor in series or in parallel with the original one.

The next lemma follows from the fact that the impedance $Z(s)$ or admittance $Y(s)$ of any network that has all reactive elements of the same kind has $\operatorname{Re}(Z(j \omega))$ and $\operatorname{Re}(Y(j \omega))$ monotonic ([26, Chapter 2.2]).

Lemma 3: Any network that has all reactive elements of the same kind can only realise regular immittances.

Lemma 4: Any network that has a path between the two external terminals 1 and $1^{\prime}$ or a cut set ([27]) that places 1 and $1^{\prime}$ in different connected parts consisting of one type of reactive element can only realise regular immittances.

We now focus attention on biquadratic positive-real functions

$$
Z(s)=\frac{A s^{2}+B s+C}{D s^{2}+E s+F}
$$


where $A, B, C, D, E, F \geq 0$. It is well known [14], [28], [29] that $Z(s)$ is positive real if and only if

$$
\sigma=B E-(\sqrt{A F}-\sqrt{C D})^{2} \geq 0 .
$$

We will make use of the resultant of the numerator and denomerator in (1) which is given by

$$
K=(A F-C D)^{2}-(A E-B D)(B F-C E) .
$$

Lemma 5: A positive-real biquadratic impedance (1) is regular if and only if the conditions of at least one of the following four cases are satisfied:

Case 1. $A F-C D \geq 0$ and

$$
\lambda_{1}=E(B \bar{F}-C E)-F(A F-C D) \geq 0,
$$

Case 2. $A F-C D \geq 0$ and

$$
\lambda_{2}=B(A E-B D)-A(A F-C D) \geq 0,
$$

Case 3. $A F-C D \leq 0$ and

$$
\lambda_{3}=D(A F-C D)-E(A E-B D) \geq 0,
$$

Case 4. $A F-C D \leq 0$ and

$$
\lambda_{4}=C(A F-C D)-B(B F-C E) \geq 0 .
$$

Lemma 6: A positive-real biquadratic impedance (1) with $Z(0)=Z(\infty) \neq 0, \infty$ (which implies $A F-C D=0$ ) is regular.

Lemma 7: A positive-real biquadratic impedance (1) with the resultant $K \leq 0$ has $\operatorname{Re}(Z(j \omega))$ and $\operatorname{Re}(Y(j \omega))$ monotonic, hence is regular.

Lemma 8: A positive-real biquadratic impedance (1) with any of the parameters $A, B, C, D, E$ and $F$ equals zero is regular. Moreover, any such impedance can be realised by a series-parallel network with at most two reactive elements and two resistive elements.

\section{A CANONICAL FORM FOR BIQUADRATICS AND NETWORK QUARTETS}

The classification of networks is facilitated by the following transformations on the impedance $Z(s)$ :

1) Multiplication by a constant multiplier $\alpha$,

2) Frequency scaling: $s \rightarrow \beta s$,

3) Frequency inversion: $s \rightarrow s^{-1}$,

4) Impedance inversion: $Z \rightarrow Z^{-1}$.

Based on Lemma 8 , it is clear that we can restrict further analysis of the synthesis problem for biquadratics (1) to the case that $A, B, C, D, E, F>0$. Using the first two transformations, $Z(s)$ can be reduced to a canonical form

$$
Z_{c}(s)=\frac{s^{2}+2 U \sqrt{W} s+W}{s^{2}+(2 V / \sqrt{W}) s+1 / W},(U, V, W>0) .
$$

For the realisation of the biquadratic, it is sufficient to restrict attention to the class in (3). This canonical form was first considered in [18].

Next we observe that frequency inversion $\left(s \leftrightarrow s^{-1}\right)$ corresponds to the transformation $W \leftrightarrow W^{-1}$ in the canonical form and impedance inversion (duality) corresponds to the transformations: $U \leftrightarrow V, W \leftrightarrow W^{-1}$. This is illustrated in Fig. 1 where the four networks $\mathrm{N}_{a}, \mathrm{~N}_{b}, \mathrm{~N}_{c}, \mathrm{~N}_{d}$ are related by interchange of inductors and capacitors (denoted by $s \leftrightarrow$

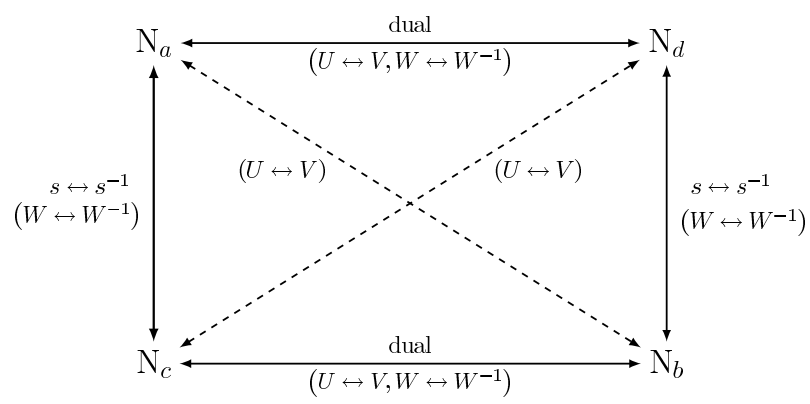

Fig. 1. Transformations relating members of a network quartet and corresponding transformation in the canonical form (3).

$s^{-1}$ ) and duality. Such a family of 4 related networks has appeared in [22] with the terminology "Untergruppe".

It follows from (2) that $Z_{c}(s)$ is positive real if and only if

$$
\sigma_{c}=4 U V+2-\left(\frac{1}{W}+W\right) \geq 0 .
$$

The resultant of $Z_{c}(s)$ is

$$
K_{c}=4 U^{2}+4 V^{2}-4 U V\left(\frac{1}{W}+W\right)+\left(\frac{1}{W}-W\right)^{2} .
$$

For any polynomial $\rho(U, V, W)$ we introduce the notation $\rho^{*}(U, V, W)=\rho\left(U, V, W^{-1}\right)$ and $\rho^{\dagger}(U, V, W)=$ $\rho(V, U, W)$. We observe that $\sigma_{c}^{*}=\sigma_{c}^{\dagger}=\sigma_{c}$ and $K_{c}^{*}=$ $K_{c}^{\dagger}=K_{c}$. Let

$$
\lambda_{c}=4 U V-4 V^{2} W-\left(\frac{1}{W}-W\right) .
$$

We can now restate Lemmas 5-7 for the canonical form (3):

Lemma 9: Let $Z_{c}(s)$ be a positive-real biquadratic defined in (3). Then:

1) $Z_{c}(s)$ is regular if and only if at least one of the following four conditions is satisfied:

Case $1 . W \leq 1$ and $\lambda_{c} \geq 0$,

Case 2. $W \leq 1$ and $\lambda_{c}^{\dagger} \geq 0$,

Case 3. $W \geq 1$ and $\lambda_{c}^{*} \geq 0$,

Case 4. $W \geq 1$ and $\lambda_{c}^{* \dagger} \geq 0$.

2) When $W=1, Z_{c}(s)$ is regular.

3) When $K_{c} \leq 0, Z_{c}(s)$ is regular.

Lemma 9-1) defines the regular region of a biquadratic in the $(U, V)$-plane, as shown in Fig. 2. In [23] the following theorem was shown.

Theorem 1: A biquadratic impedance (1) can be realised by series-parallel five-element networks with two reactive elements if and only if it is regular.

\section{Five-Element Three-Reactive Element SYNTHESIS OF THE BIQUADRATIC}

In [17], Ladenheim claims there are eight series-parallel networks which can realise classes of biquadratic immittances which otherwise would require a full Bott-Duffin synthesis. Vasiliu [19] later shows that four of these networks can be realised by networks of two reactive and three resistive elements. In [17], [19] it is not shown whether the remaining four networks can realise biquadratic immittances 


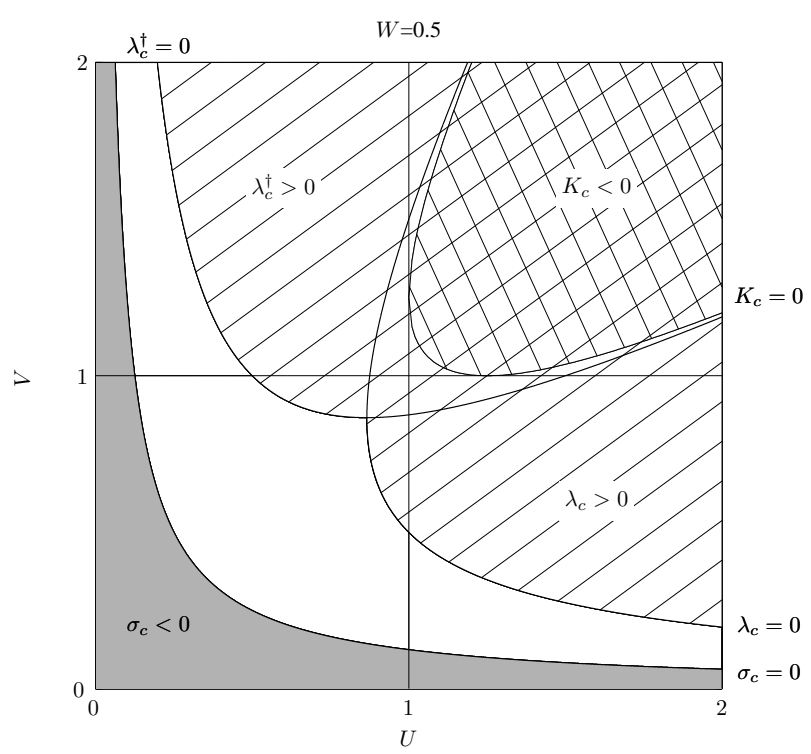

Fig. 2. The regular impedances of (3) with $W \in(0,1)$.

which cannot be realised in a simpler manner. Here we provide the range of parameter values corresponding to non-regular biquadratics in the canonical form which are realisable by this network quartet (Theorem 3 and Table I).

Theorem 2: All series-parallel networks with three reactive and two resistive elements can only realise regular immittances except for the network quartet of Fig. 3.

Proof: The procedure used to reduce to eight seriesparallel networks is not described in [17]. A complete elimination process based on the regularity concept is given in [30].

It can be calculated that the positive real biquadratic impedance (1) with all parameters positive and the resultant $K \neq 0$ can be realised as in Fig. 3(a) with $R_{1}, R_{2}, L_{1}, C_{1}$ and $C_{2}$ positive and finite, if and only if

$$
D(B E-C D)^{2}-A E^{2}(B E-C D)+A^{2} E^{2} F=0
$$

(the extra inequality $B E-C D>0$ given in [17] for this network can be seen to be redundant). The realisability conditions for the other three networks can be obtained by the transformations $s \leftrightarrow s^{-1}, Z \leftrightarrow Z^{-1}$. The realisability condition of the network Fig. 3(a) for the canonical form (3) can be derived from (4), which is:

$$
\begin{array}{r}
\gamma_{3}=\gamma_{3+} \gamma_{3-}=16 U^{2} W^{2} V^{2}-8 U W\left(2 U^{2} W^{2}+1\right) V \\
+4 U^{2} W^{2}+4 U^{2} W^{4}+1=0
\end{array}
$$

where

$$
\gamma_{3 \pm}=4 V W U-2 W^{2}\left(U^{2} \pm U \sqrt{U^{2}-1}\right)-1 .
$$

It is interesting to note that a necessary condition for realisability in this quartet is that $U \geq 1$ or $V \geq 1$. We now describe explicitly the non-regular realisable regions in the $(U, V)$-plane for this quartet.

Theorem 3: The impedance (3) can be realised by the network shown in Fig. 3(a) (Fig. 3(b)) if and only if $(U, V)$
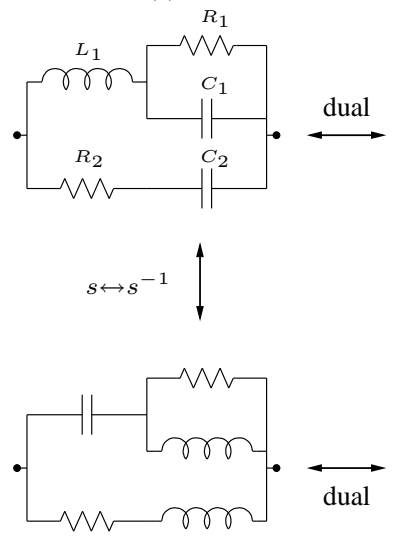

(c) (d)
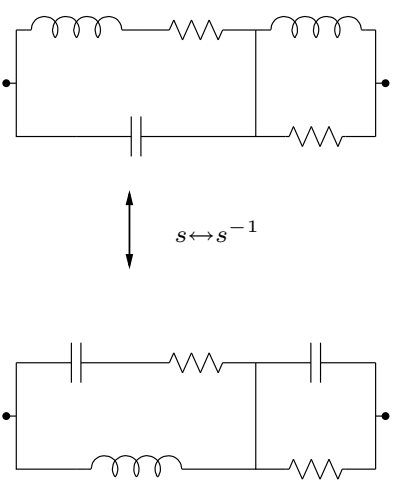

(b)
Fig. 3. The series-parallel three-reactive five-element network quartet that can realise non-regular biquadratics.

TABLE I

THE REALISABILITY CONDITIONS OF THE NETWORK QUARTET OF FIG. 3 FOR A BIQUADRATIC IMPEDANCE IN THE CANONICAL FORM (3).

\begin{tabular}{|l|l|l|}
\hline Networks & $\begin{array}{l}\text { Necessary and Suffi- } \\
\text { cient conditions for a } \\
\text { non-regular (3) to be } \\
\text { realisable }\end{array}$ & $\begin{array}{l}\text { Range of } W \text { for } \\
\text { which } \\
\text { region is non-empty } \\
\text { non-regular }\end{array}$ \\
\hline Fig. 3(a) & $\gamma_{3}=0$ & $W \in(0,0.3702)$ \\
\hline Fig. 3(b) & $\gamma_{3}^{\dagger}=0$ & $W \in(0,0.3702)$ \\
\hline Fig. 3(c) & $\gamma_{3}^{*}=0$ & $W \in(1 / 0.3702, \infty)$ \\
\hline Fig. 3(d) & $\gamma_{3}^{* \dagger}=0$ & $W \in(1 / 0.3702, \infty)$ \\
\hline
\end{tabular}

is on the curve $\gamma_{3}=0\left(\gamma_{3}^{\dagger}=0\right)$. Furthermore, (3) can only be non-regular when $W \in(0,0.3702)$.

Proof: See [31].

The conditions for a non-regular biquadratic impedance in the canonical form (3) to be realisable by the network quartet of Fig. 3 are summarised in Table I.

\section{SiX-Element Three-Reactive Element SYNTHESIS OF THE BIQUADRATIC}

Vasiliu [20] claims that, there are in total 16 series-parallel networks with three reactive elements, which can realise biquadratic immittances which otherwise would require a full Bott-Duffin synthesis. In this section, a restudy of this topic based on the concept of regular positive-real functions is presented. We go beyond [20] to identify the non-regular realisable regions in the $(U, V)$-plane for each quartet. We will show that the non-regular realisable regions are identical for three of the quartets.

\section{A. Elimination of the Networks that Can Only Realise Reg- ular Biquadratics}

Lemma 10: The network shown in Fig. 4 can only realise regular biquadratic impedances (1).

Proof: See [32].

Lemma 11: The networks shown in Fig. 5 can only realise regular biquadratic impedances (1). 


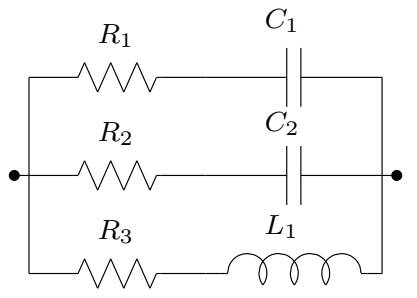

Fig. 4. The series-parallel six-element network with three reactive elements which based on Lemma 10 can only realise regular biquadratics.

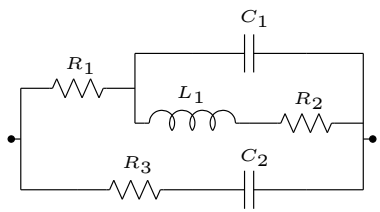

(a)

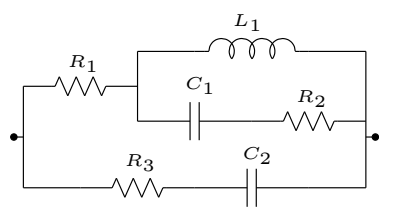

(b)

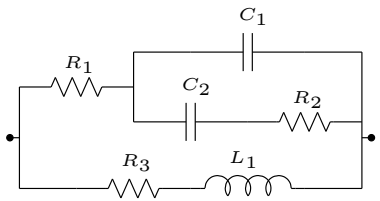

(c)

Fig. 5. Three series-parallel six-element networks with three reactive elements which based on Lemma 11 can only realise regular biquadratics.

Proof: See [32].

Theorem 4: A non-regular biquadratic immittance can be realised by a series-parallel network with three reactive and three resistive elements if and only if it is realisable by some network in the four network quartets of Fig. 6.

Proof: The proof makes use of Lemmas 10, 11 and the properties of regularity introduced in Section II. The detailed proof is provided in [30].

B. The Non-Regular Realisable Region of the Network Quartet Fig. 6-I, II, III

By the method introduced in [20], the realisability conditions of the network Fig. 6-I(a) for (1) can be derived, which are:

$$
B^{2}-4 C A \geq 0
$$

and

$$
C(E-C \mu)^{2}>F(B(E-C \mu)-A F)>0,
$$

where

$$
\mu=\frac{D\left(B \pm \sqrt{B^{2}-4 A C}\right)}{2 A C} .
$$

It can be calculated that $\lambda_{c}=0\left(\lambda_{c}^{*}=0\right)$ is equivalent to either $\lambda_{c-}=0$ or $\lambda_{c+}=0\left(\lambda_{c-}^{*}=0\right.$ or $\left.\lambda_{c+}^{*}=0\right)$, where

$$
\lambda_{c \pm}=2 V W-\left(U \pm \sqrt{U^{2}-1+W^{2}}\right) .
$$

Lemma 12: Any $(U, V, W)$ satisfying $W<1, U \geq 1$, $\lambda_{c-}>0\left(W>1, U \geq 1, \lambda_{c-}^{*}>0\right)$ is inside the regular region.

Proof: See [32].

Theorem 5: A non-regular biquadratic impedance (3) is realisable by the network of Fig. 6-I(a) if and only if $U \geq 1$,

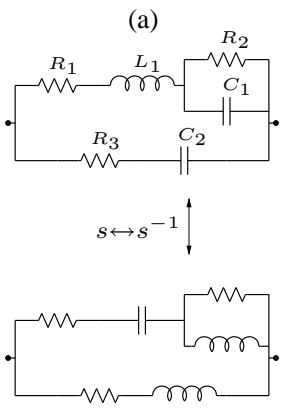

(c) (d)

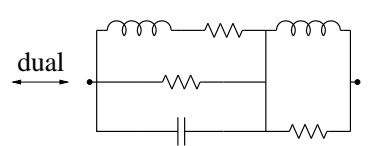

I

$s \leftrightarrow s^{-1}$

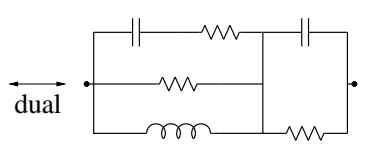

(b)
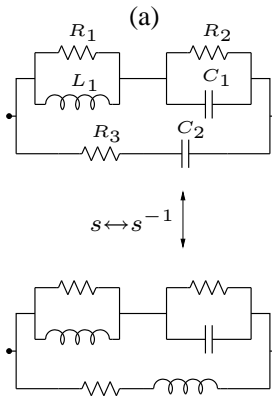

(c) (d)
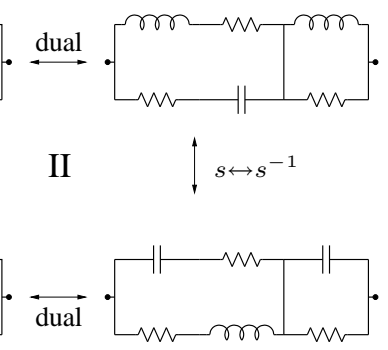

(b)

(d)

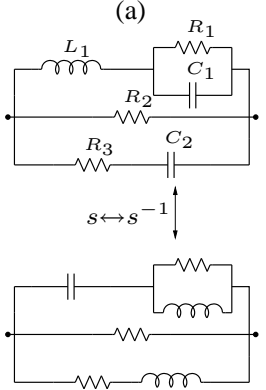

(c)
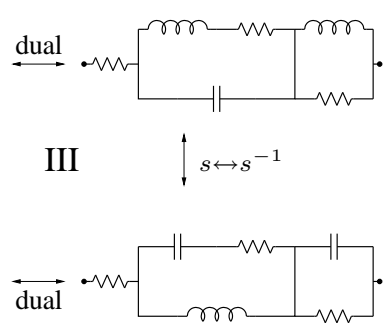

(b)

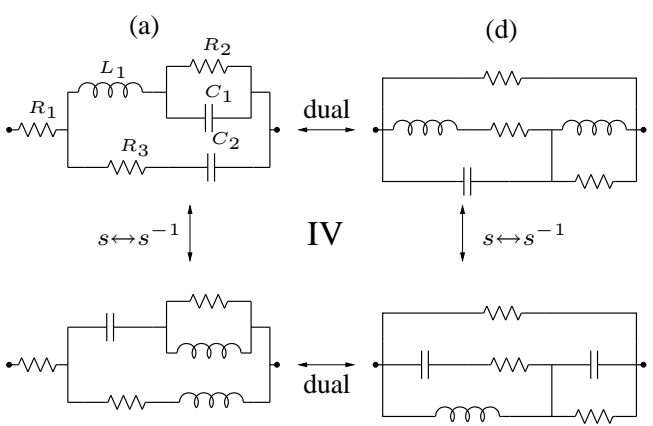

(c) (b)
Fig. 6. The four network quartets of the series-parallel three-reactive sixelement networks that can realise non-regular biquadratics. 


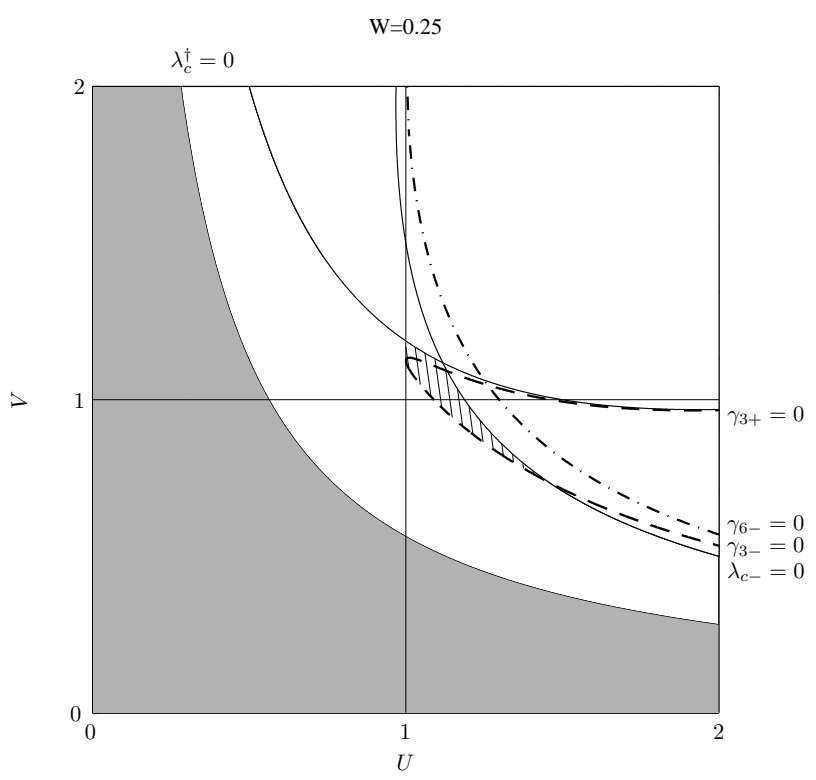

Fig. 7. The non-regular impedance (3) that can be realised by the networks of Figs. 6-I(a), II(a) and III(a) with $W \in(0,0.3702)$.

$\gamma_{3-}>0$. (See hatched region of Fig. 7.) Moreover, this nonregular region is non-empty if and only if $W \in(0,0.3702)$.

Proof: We first rewrite the realisability conditions for the network of Fig. 6-I(a) for the canonical form (3). Note that (5) is equivalent to $U \geq 1$ and the right hand inequality in (6) is equivalent to $\gamma_{3-}>0$ or $\gamma_{3+}>0$, depending on whether the - or + sign is chosen for $\mu$ in (7).

When $W<1$, we first consider the case that negative sign is chosen for $\mu$. The left hand inequality in (6) is equivalent to $\gamma_{6}=\gamma_{6+} \gamma_{6-}>0$, where

$\gamma_{6 \pm}=2 V W-W^{2}\left(U-\sqrt{U^{2}-1}\right)-\left(U \pm \sqrt{U^{2}-1}\right)$,

which is equivalent to $U \geq 1,(U, V)$ is above $\gamma_{6+}=0$ or below $\gamma_{6-}=0$. It can be calculated that the resultant of the polynomials $\gamma_{6-}$ and $\lambda_{c-}$ is

$2 W\left(W^{2}\left(\sqrt{U^{2}-1}-U\right)+\sqrt{U^{2}-1}-\sqrt{U^{2}-1+W^{2}}\right)$

which cannot equal zero. Further, it can be checked that the curve $\gamma_{6-}=0$ is always above the curve $\lambda_{c-}=0$ (see Fig. 7). Since $\gamma_{6+} \leq \gamma_{6-}$, if $(U, V)$ is in the nonregular region for $W<1, \gamma_{6}>0$ is equivalent to $\gamma_{6-}<0$ (by Lemma 12), which is then redundant. For the case that positive sign is chosen for $\mu$, since $\gamma_{3+}>0$ implies $\gamma_{3-}>0$ we do not find any additional non-regular region in the $(U, V)$-plane. Based on Theorem 3 we know that $\gamma_{3-}=0$ can be outside of the regular region with $W<1$ if and only if $W \in(0,0.3702)$. This proves the result for the case $W<1$.

When $W>1$, it follows from the proof of Theorem 3 that the curves $\gamma_{3 \pm}=0$ are inside the regular region $\lambda_{c}^{*}>0$. Then based on Lemma 12, neither $\gamma_{3-}>0$ nor $\gamma_{3+}>0$ covers any non-regular region with $W>1$.

The realisability conditions for the biquadratic (1) for the networks of Figs. 6-II(c) and III(c) are given in [20]. It can
TABLE II

THE REALISABILITY CONDITIONS OF THE NETWORK QUARTETS OF FIG. 6-I,II,III FOR A BIQUADRATIC IMPEDANCE IN THE CANONICAL FORM (3).

\begin{tabular}{|l|l|l|}
\hline Networks & $\begin{array}{l}\text { Nec. and Suff. } \\
\text { conditions for a } \\
\text { non-regular (3) to } \\
\text { be realisable }\end{array}$ & $\begin{array}{l}\text { Range of } W \text { for which } \\
\text { non-regular region is non- } \\
\text { empty }\end{array}$ \\
\hline Fig. 6-I,II,III(a) & $U \geq 1, \gamma_{3-}>0$ & $W \in(0,0.3702)$ \\
\hline Fig. 6-I,II,III(b) & $V \geq 1, \gamma_{3-}^{\dagger}>0$ & $W \in(0,0.3702)$ \\
\hline Fig. 6-I,II,III(c) & $U \geq 1, \gamma_{3-}^{*}>0$ & $W \in(1 / 0.3702, \infty)$ \\
\hline Fig. 6-I,II,III(d) & $V \geq 1, \gamma_{3-}^{* \dagger}>0$ & $W \in(1 / 0.3702, \infty)$ \\
\hline
\end{tabular}

TABLE III

THE REALIS ABILITY CONDITIONS OF THE NETWORK QUARTET OF FIG. 6-IV FOR A BIQUADRATIC IMPEDANCE IN THE CANONICAL FORM (3).

\begin{tabular}{|l|l|}
\hline Networks & Realisability Conditions \\
\hline Fig. 6-IV(a) & $\left\{(U, V) \mid \exists x \in\left(0, \min \left(1, W^{2}, \frac{U W}{V}\right)\right): f_{3}(x)=0\right\}$ \\
\hline Fig. 6-IV(b) & $\left\{(U, V) \mid \exists x \in\left(0, \min \left(1, W^{2}, \frac{V W}{U}\right)\right): f_{3}^{\dagger}(x)=0\right\}$ \\
\hline Fig. 6-IV(c) & $\left\{(U, V) \mid \exists x \in\left(0, \min \left(1, \frac{1}{W^{2}}, \frac{U}{V W}\right)\right): f_{3}^{*}(x)=0\right\}$ \\
\hline Fig. 6-IV(d) & $\left\{(U, V) \mid \exists x \in\left(0, \min \left(1, \frac{1}{W^{2}}, \frac{V}{U W}\right)\right): f_{3}^{* \dagger}(x)=0\right\}$ \\
\hline
\end{tabular}

be checked that the realisable region in the $(U, V)$-plane for the canonical form (3) of the network quartets of Figs. 6-II and III differ and are also different from the network quartet of Fig. 6-I. However it turns out that the set of non-regular biquadratics which are realisable by these network quartets is the same. The conditions for a non-regular biquadratic impedance (3) to be realisable by the network quartets of Fig. 6-I, II, III are summarised in Table II.

\section{The Non-Regular Realisable Region of the Network Quar- tet Fig. 6-IV}

For any polynomial $f(x, U, V, W)$ we introduce the notation $f^{*}(x, U, V, W)=f\left(x, U, V, W^{-1}\right)$, and $f^{\dagger}(x, U, V, W)=f(x, V, U, W)$. The complete realisability conditions of the network quartet of Fig. 6-IV for the biquadratic canonical form (3) can be deduced from [20]. These are summarised in Table III, where $f_{3}(x)=x^{3}+l_{1} x^{2}+l_{2} x-\gamma_{3}=0$, and

$$
\begin{array}{r}
l_{1}=12 V^{2}-16 V^{4}-3-4 V^{2} W^{2}+16 U V^{3} W-8 V U W, \\
l_{2}=8 U^{2} W^{2}-8 U V W\left(4 V U W-W^{2}+1-4 V^{2}\right) \\
-8 V^{2}+3 .
\end{array}
$$

The conditions of Table III can be made more explicit using the method of sturm chains, though the polynomials in the sturm sequence are quite complex. Fig. 8 shows the realisable regions in the $(U, V)$-plane when $W$ equals 0.35 for the network of Fig. 6-IV(a). Part of the boundary curve for the non-regular realisable region for the network of Fig. 6-IV(a) is determined by the zero set of a high order 


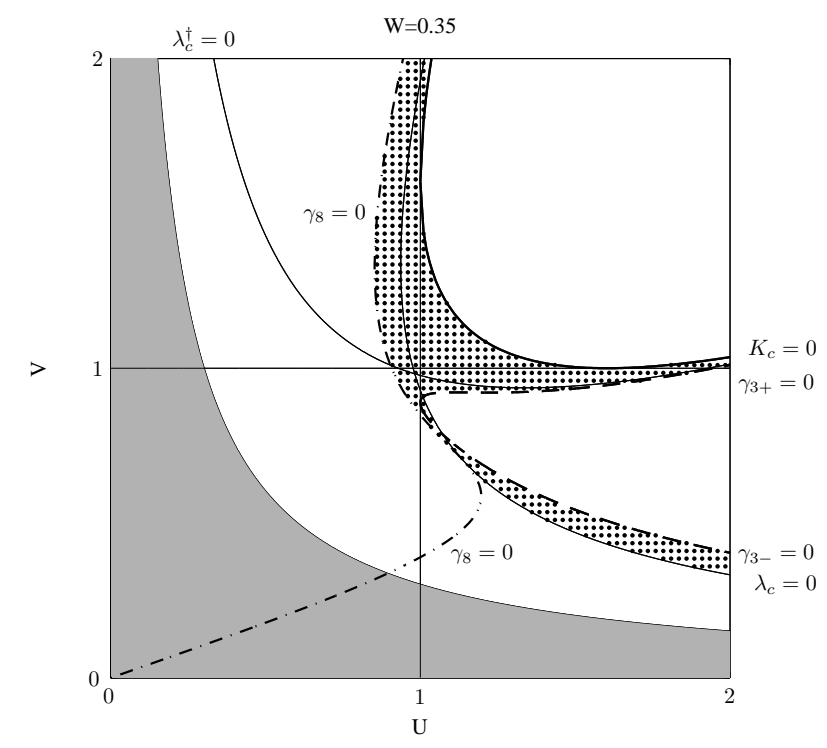

Fig. 8. The whole region in the $(U, V)$-plane that can be realised by the network of Fig. 6-IV(a) with $W=0.35$.

polynomial $\gamma_{8}$ involving $U, V$ and $W$. It is to be noted that the non-regular regions which are realisable by the quartet of Fig. 6-IV are different from the previous three. The full characterisation of the realisable regions for this quartet are given in [32].

\section{CONCLUSION}

This paper studies five- and six-element series-parallel two-terminal networks with three reactive elements and presents a complete characterisation and graphical representation of the realisable conditions for these networks. It is shown that the non-regular realisable regions for three of the six-element network quartets are identical and have a boundary which coincides with the realisability curve for the five-element quartet. The fourth six-element quartet is shown to realise a different non-regular region from the other quartets.

\section{REFERENCES}

[1] R. Bott and R. J. Duffin, "Impedance synthesis without use of transformers," J. Appl. Phys., vol. 20, p. 816, 1949.

[2] R. H. Pantell, "A new method of driving point impedance synthesis," Proc. IRE, vol. 42, p. 861, 1954.

[3] F. M. Reza, "A supplement to brune synthesis," Commun. and Electronics., pp. 85-90, March 1955.

[4] A. Fialkow and I. Gerst, "Impedance synthesis without mutual coupling," Quart. Appl. Math., vol. 12, pp. 420-422, 1955.

[5] S. Seshu, "Minimal realizations of the biquadratic minimum function," IRE Transactions on Circuit Theory, pp. 345-350, Dec. 1959.

[6] M. C. Smith, "Synthesis of mechanical networks: the inerter," IEEE Trans. Automatic Control, vol. 47, no. 10, pp. 1648-1662, 2002.

[7] C. Papageorgiou and M. C. Smith, "Positive real synthesis using matrix inequalities for mechanical networks: application to vehicle suspension," IEEE Trans. on Contr. Syst. Tech., vol. 14, pp. 423-435, 2006.

[8] M. C. Smith and F.-C. Wang, "Performance benefits in passive vehicle suspensions employing inerters," Vehicle System Dynamics, vol. 42, pp. 235-237, 2004.
[9] S. Evangelou, D. J. Limebeer, R. S. Sharp, and M. C. Smith, "Control of motorcycle steering instabilities - passive mechanical compensators incorporating inerters," IEEE Control Systems Magazine, pp. 78-88, October 2006.

[10] —, "Mechanical steering compensation for high-performance motorcycles," Transactions of ASME, J. of Applied Mechanics, vol. 74, pp. 332-346, 2007.

[11] Cambridge University Engineering Department news item, 19 August 2008. [Online]. Available: http://www.eng.cam.ac.uk/news/stories/ 2008/McLaren/

[12] E. L. Ladenheim, "A synthesis of biquadratic impedances," Master's thesis, Polytechnic Inst. of Brooklyn, N. Y., 1948.

[13] R. M. Foster, "Academic and theoretical aspects of circuit theory," in Proc. IRE, vol. 50, May 1962, pp. 866-871.

[14] R. M. Foster and E. L. Ladenheim, "A class of biquadratic impedances," IEEE Trans. on Circuit Theory, pp. 262-265, 1963.

[15] R. M. Foster, "Biquadratic impedances realisable by a generalization of the five-element minimum-resistance bridges," IEEE Trans. on Circuit Theory, vol. CT-10, pp. 363-367, September 1963.

[16] E. L. Ladenheim, "A special biquadratic structure," IEEE Trans. on Circuit Theory, pp. 455-456, 1963.

[17] — , "Three-reactive five-element biquadratic structures," IEEE Trans. on Circuit Theory, pp. 88-97, 1964.

[18] M. Reichert, "Die kanonisch und übertragerfrei realisierbaren Zweipolfunktionen zweiten Grades (transformerless and canonic realisation of biquadratic immittance functions)," Arch. Elek. Übertragung, vol. 23, pp. 201-208, Apr. 1969

[19] C. G. Vasiliu, "Three-reactive five-element structures," IEEE Trans. Circuit Theory, vol. CT-16, p. 99, Feb. 1969.

[20] — " "Series-parallel six-element synthesis of the biquadratic impedances," IEEE Trans. on Circuit Theory, pp. 115-121, 1970

[21] — "Correction to "series-parallel six-element synthesis of the biquadratic impedances',” p. 207, November 1970.

[22] G. Dittmer, "Zur realisierung von RLC-Brückenzweipolen mit zwei Reaktanzen und mehr als drei Widerständen (on the realisation of RLC two-terminal bridge networks with two reactive and more than three resistive elements)," Nachrichtentechnische Zeitschrift, vol. 23, pp. 225-230, 1970.

[23] J. Z. Jiang and M. C. Smith, "Regular positive-real functions and passive networks comprising two reactive elements." European Control Conference (ECC), August 2009.

[24] P. M. Lin, "A theorem on equivalent one-port networks," IEEE Trans. on Circuit Theory, vol. CT-12, pp. 619-621, Dec. 1965.

[25] — "On biquadratic impedances with two reactive elements," IEEE Trans. on Circuit Theory, vol. CT-18, p. 277, Mar. 1971.

[26] E. A. Guillemin, Synthesis of Passive Networks. John Wiley \& Sons, 1957.

[27] S. Seshu and M. B. Reed, Linear Graphs and Electrical Networks. Addison-Wesley, 1961.

[28] M. E. V. Valkenburg, Introduction to Modern Network Synthesis. John Wiley \& Sons, 1960

[29] M. Z. Q. Chen and M. C. Smith, "A note on tests for positive-real functions," IEEE Transactions on Automatic Control, vol. 54, no. 2, pp. 390-393, 2009.

[30] J. Z. Jiang and M. C. Smith, "Regular positive-real functions and the classification of transformerless series-parallel networks," in Perspectives in Mathematical System Theory, Control, and Signal Processing. Springer Verlag, to appear.

[31] _ , "Regular positive-real functions and five-element network realisations for electrical and mechanical networks," in preparation.

[32] _ _ "Synthesis of positive-real functions with low-complexity seriesparallel networks," in preparation. 